\title{
POLYHEDRAL ANALYSIS FOR THE UNCAPACITATED HUB LOCATION PROBLEM WITH MODULAR ARC CAPACITIES*
}

\author{
HANDE YAMAN ${ }^{\dagger}$
}

\begin{abstract}
We consider the problem of installing a two-level telecommunication network. Terminal nodes communicate with each other through hubs. Hubs can be installed on terminal nodes and they are interconnected by a complete network. Each terminal is connected directly to a hub node. Integer amounts of capacity units are installed on the arcs between hub pairs and terminals and their hubs. The aim is to minimize the cost of installing hubs and capacity units on arcs. We present valid and facet defining inequalities for the polyhedron associated with this problem.
\end{abstract}

Key words. hub location, polyhedral analysis, lifting

AMS subject classifications. 90C10, 90C57, 90B 80

DOI. $10.1137 /$ S0895480103439157

1. Introduction. We consider the problem of locating hubs in a telecommunication network. Hubs (servers, concentrators, etc.) are installed to route the traffic of terminals (users). Given a set of terminals, a subset is chosen to be the set of hub locations. Each terminal that does not become a hub is directly connected to a single hub. The network connecting the hubs is called the backbone network and a network connecting the terminals to a hub is called a local access network (LAN). We consider telecommunication networks where the backbone is complete and the LANs are stars.

The traffic between two terminals goes from the origin terminal to its hub, then to the hub of the destination terminal, and then to the destination itself. So the total traffic on the arc from a terminal to its hub is the traffic originating at that terminal node, and the traffic on the arc from a hub to a terminal connected to that hub is the traffic arriving at that terminal node. The total traffic to travel from hub $j$ to hub $l$ is the traffic from terminals connected to hub $j$ to terminals connected to hub $l$. The traffic flows on arcs and capacity units can be installed on arcs in integer amounts.

In Figure 1.1, we see a network with three hubs. The traffic between any two nodes is 0.5 and the capacity unit is 1 on all arcs. The amount of capacity units to be installed on the arcs are given in the figure. For example, we need to install $\lceil 7 \times 0.5\rceil=4$ capacity units on an arc from a terminal to its hub.

The cost of installing such a telecommunication network is the sum of the cost of locating hubs and the cost of installing capacity units on arcs. The uncapacitated hub location problem with modular arc capacities (HLM) is the problem of locating hubs and connecting the remaining nodes to hubs with the aim of minimizing this total cost. Labbé and Yaman [11] prove that the special case of HLM where the cost of installing capacity units on the backbone network is zero is NP-hard.

Campbell, Ernst, and Krishnamoorthy [3] give a survey of hub location problems. Klincewicz [7] gives a survey of hub location problems in telecommunications.

Very little is known about the polyhedra associated with hub location problems. A similar problem with no cost for installing capacity units on arcs but a cost for routing

\footnotetext{
* Received by the editors December 30, 2003; accepted for publication (in revised form) January 3, 2005; published electronically November 4, 2005.

http://www.siam.org/journals/sidma/19-2/43915.html

${ }^{\dagger}$ Bilkent University, Department of Industrial Engineering, Bilkent, 06800 Ankara, Turkey (hyaman@bilkent.edu.tr).
} 


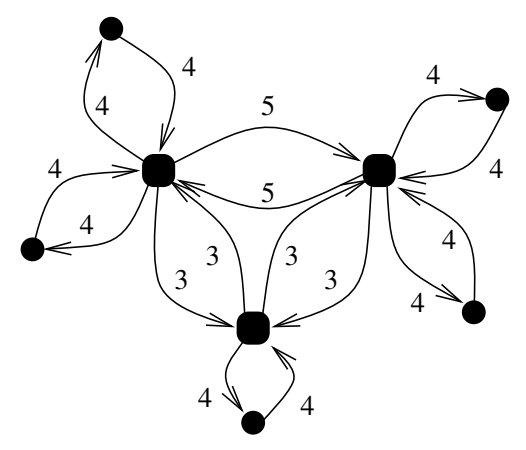

FIG. 1.1. A network with three hubs.

the traffic is called the uncapacitated hub location problem with single assignment (HLs). Polyhedral analysis for this problem can be found in [12] and [10]. If, in addition, we allow a terminal to be connected to several hubs, then the problem is called the uncapacitated hub location problem with multiple assignment (HLm). Polyhedral properties of HLm are studied by Hamacher et al. [6].

Chung, Myung, and Tcha [5] study a version of HLM where there is a fixed cost of establishing a link between two hubs. In HLM, this corresponds to the case where backbone links are uncapacitated, meaning that if two nodes become hubs, only one capacity unit is installed between them. The authors propose a branch and cut algorithm for this problem.

Yaman and Carello [15] consider a generalization of HLM where hubs are capacitated; the amount of traffic transiting through a hub is limited by the capacity of the hub. They present a metaheuristic and a branch and cut algorithm to solve this problem. Their branch and cut algorithm uses cuts given in [12] and [10].

In this paper, we present valid and facet defining inequalities for the polyhedron associated with HLM. We give several lifting results which can be used to derive further facet defining inequalities. The paper is organized as follows. In section 2, we give a formulation of the problem. We present valid inequalities in section 3. Section 4 is devoted to polyhedral analysis. We conclude in section 5 .

2. Formulation. Let $I$ denote the set of terminal nodes with $|I|=n$. Any distinct pair of terminal nodes defines a commodity. We denote by $K$ the set of commodities. For commodity $(i, m) \in K, i$ is the origin, $m$ is the destination, and $t_{i m}$ is the amount of traffic to be routed from $i$ to $m$. We define $t_{i i}$ to be 0 for all $i \in I$.

Each terminal either becomes a hub or is connected to another node which becomes a hub. The cost of installing a hub at node $i \in I$ is denoted by $C_{i i}$. Hubs are connected by a complete directed graph. Each nonhub node is directly connected to its hub. Integer amounts of capacity are installed on the arcs between pairs of hubs and between terminals and their hubs. We assume that the capacity unit on all arcs is 1 and that the demands are scaled accordingly. The capacity of each terminal-hub and hub-terminal arc is fully determined by the chosen terminal-hub connection. The cost of connecting node $i \in I$ to node $j \in I \backslash\{i\}$, denoted by $C_{i j}$, is equal to the cost of installing $\left\lceil\sum_{m \in I} t_{i m}\right\rceil+\left\lceil\sum_{m \in I} t_{m i}\right\rceil$ capacity units between nodes $i$ and $j$.

We define the arc set $A=\{(j, l): j \in I, l \in I, j \neq l\}$. We denote by $R_{j l}$ the cost of installing a capacity unit on arc $(j, l)$ if it becomes a backbone arc. Let $K_{j l}^{\prime}$ be the set of commodities $(i, m)$ such that $i$ is connected to $j$ and $m$ is connected to 
$l$. If nodes $j$ and $l$ become hubs, then the amount of flow on $\operatorname{arc}(j, l)$ is given by $\sum_{(i, m) \in K_{j l}^{\prime}} t_{i m}$ and $\left\lceil\sum_{(i, m) \in K_{j l}^{\prime}} t_{i m}\right\rceil$ units of capacity should be installed on this arc.

We define the assignment variable $x_{i j}$ to be 1 if terminal $i \in I$ is assigned (connected) to hub $j \in I$ and 0 otherwise. If node $i$ becomes a hub, then $x_{i i}$ is 1 . We further define $z_{j l}$ to be the amount of capacity units installed on $\operatorname{arc}(j, l) \in A$.

The HLM can be formulated as follows (see [12]):

$$
\begin{aligned}
& \min \sum_{i \in I} \sum_{j \in I} C_{i j} x_{i j}+\sum_{(j, l) \in A} R_{j l} z_{j l} \\
& \text { subject to } \sum_{j \in I} x_{i j}=1 \quad \forall i \in I \text {, } \\
& x_{i j} \leq x_{j j} \quad \forall(i, j) \in A, \\
& z_{j l} \geq \sum_{(i, m) \in K^{\prime}} t_{i m}\left(x_{i j}+x_{m l}-1\right) \quad \forall(j, l) \in A, K^{\prime} \subseteq K, \\
& z_{j l} \text { integer } \quad \forall(j, l) \in A \text {, } \\
& x_{i j} \in\{0,1\} \quad \forall i \in I, j \in I .
\end{aligned}
$$

Constraints (2.2), (2.3), and (2.6) ensure that each terminal either becomes a hub or is assigned to exactly one hub. Constraints (2.4) relate the capacity vector $z$ to the assignment vector $x$. For $\operatorname{arc}(j, l) \in A$, because of constraints (2.5) and (2.6), constraint set (2.4) is equivalent to

$$
z_{j l} \geq\left\lceil\max _{K^{\prime} \subseteq K}\left(\sum_{(i, m) \in K^{\prime}} t_{i m}\left(x_{i j}+x_{m l}-1\right)\right)\right\rceil=\left\lceil\sum_{(i, m) \in K_{j l}^{\prime}} t_{i m}\right\rceil .
$$

If $R_{j l}>0$, then an optimal solution satisfies the inequality at equality.

The objective function (2.1) consists of the cost of locating hubs and the cost of installing capacity units on arcs.

3. Valid inequalities. In this section, we present families of valid inequalities for the polyhedron associated with HLM and point out the domination relations among these valid inequalities. We investigate inequalities that involve both the assignment and the capacity variables.

Definition 3.1. Let

$$
F=\left\{(x, z) \in\{0,1\}^{n^{2}} \times \mathbb{Z}^{n(n-1)}:(x, z) \text { satisfies }(2.2)-(2.6)\right\}
$$

and

$$
P=\operatorname{conv}(F) .
$$

Labbé, Yaman, and Gourdin [12] study the HLs which is obtained by relaxing integrality constraints (2.5) in HLM. They derive valid inequalities by projecting out the flow variables in a larger formulation for this relaxed problem. These inequalities are given in the following proposition.

Proposition 3.2 (Labbé, Yaman, and Gourdin [12]). Let $S$ and $T$ be nonempty disjoint subsets of $I$ and $K^{\prime} \subseteq K$. The projection inequality

$$
\sum_{j \in S} \sum_{l \in T} z_{j l} \geq \sum_{(i, m) \in K^{\prime}} t_{i m}\left(\sum_{j \in S} x_{i j}+\sum_{l \in T} x_{m l}-1\right)
$$


is valid for $P$.

Constraints (2.4) are projection inequalities where sets $S$ and $T$ are singletons.

Projection inequalities (3.1) ignore the integrality of $z_{j l}$ variables. Now we present a family of inequalities which use this information.

For $K^{\prime} \subseteq K$, let

$$
O\left(K^{\prime}\right)=\left\{i \in I: \exists m \in I \backslash\{i\} \text { with }(i, m) \in K^{\prime}\right\}
$$

and

$$
D\left(K^{\prime}\right)=\left\{i \in I: \exists m \in I \backslash\{i\} \text { with }(m, i) \in K^{\prime}\right\} .
$$

Proposition 3.3. Let $S$ and $T$ be nonempty disjoint subsets of $I$ and $K^{\prime} \subseteq K$. Inequality

$$
\sum_{j \in S} \sum_{l \in T} z_{j l} \geq\left\lceil\sum_{(i, m) \in K^{\prime}} t_{i m}\right\rceil\left(1-\sum_{i \in O\left(K^{\prime}\right)} \sum_{j \in I \backslash S} x_{i j}-\sum_{m \in D\left(K^{\prime}\right)} \sum_{l \in I \backslash T} x_{m l}\right)
$$

is valid for $P$.

Proof. For $(x, z) \in F$, the right-hand side of inequality $(3.2)$ is $\left\lceil\sum_{(i, m) \in K^{\prime}} t_{i m}\right\rceil$ if $\sum_{j \in I \backslash S} x_{i j}=0$ for all $i \in O\left(K^{\prime}\right)$ and $\sum_{l \in I \backslash T} x_{m l}=0$ for all $m \in D\left(K^{\prime}\right)$. It is nonpositive otherwise.

Notice that different sets $K^{\prime}$ can lead to the same sets $O\left(K^{\prime}\right)$ and $D\left(K^{\prime}\right)$. For a given fractional solution, it is important to be able to choose among these subsets $K^{\prime}$ the one which leads to the most violated inequality.

For subsets $O$ and $D$ of $I$, let

$$
\kappa(O, D)=\{(i, m) \in K: i \in O \text { and } m \in D\} .
$$

Proposition 3.4. Let $(x, z)$ be a fractional solution which satisfies constraints (2.2). If there exists an inequality (3.2) violated by $(x, z)$, then there exists a violated inequality (3.2) for some $K^{\prime} \subseteq K$ such that $O\left(K^{\prime}\right) \cap D\left(K^{\prime}\right)=\emptyset$ and $K^{\prime}=$ $\kappa\left(O\left(K^{\prime}\right), D\left(K^{\prime}\right)\right)$.

Proof. For $K^{\prime} \subseteq K$, if $\left|O\left(K^{\prime}\right) \cap D\left(K^{\prime}\right)\right| \geq 1$, then

$$
\begin{aligned}
& -\sum_{i \in O\left(K^{\prime}\right)} \sum_{j \in I \backslash S} x_{i j}-\sum_{m \in D\left(K^{\prime}\right)} \sum_{l \in I \backslash T} x_{m l} \\
= & \sum_{i \in O\left(K^{\prime}\right)} \sum_{j \in S} x_{i j}+\sum_{m \in D\left(K^{\prime}\right)} \sum_{l \in T} x_{m l}-\left|O\left(K^{\prime}\right)\right|-\left|D\left(K^{\prime}\right)\right|+1 \\
= & \sum_{i \in O\left(K^{\prime}\right) \backslash D\left(K^{\prime}\right)} \sum_{j \in S} x_{i j}-\left|O\left(K^{\prime}\right) \backslash D\left(K^{\prime}\right)\right|+\sum_{m \in D\left(K^{\prime}\right) \backslash O\left(K^{\prime}\right)} \sum_{l \in T} x_{m l}-\left|D\left(K^{\prime}\right) \backslash O\left(K^{\prime}\right)\right| \\
& \quad+\sum_{i \in O\left(K^{\prime}\right) \cap D\left(K^{\prime}\right)} \sum_{j \in S \cup T} x_{i j}-2\left|O\left(K^{\prime}\right) \cap D\left(K^{\prime}\right)\right|+1 \\
\leq & \sum_{i \in O\left(K^{\prime}\right) \cap D\left(K^{\prime}\right)} \sum_{j \in S \cup T} x_{i j}-2\left|O\left(K^{\prime}\right) \cap D\left(K^{\prime}\right)\right|+1 \\
\leq & \left(-\left|O\left(K^{\prime}\right) \cap D\left(K^{\prime}\right)\right|+1\right) \leq 0 .
\end{aligned}
$$

Therefore, inequality (3.2) for this choice of $K^{\prime}$ cannot be violated. This proves that if inequality (3.2) is violated for $K^{\prime}$, then $O\left(K^{\prime}\right) \cap D\left(K^{\prime}\right)=\emptyset$. The second part of the proposition is then trivial. 
If $S$ and $T$ are singletons, then inequality (3.2) becomes

$$
z_{j l} \geq\left\lceil\sum_{(i, m) \in K^{\prime}} t_{i m}\right\rceil\left(1-\sum_{i \in O\left(K^{\prime}\right)} \sum_{u \in I \backslash\{j\}} x_{i u}-\sum_{m \in D\left(K^{\prime}\right)} \sum_{u \in I \backslash\{l\}} x_{m u}\right) .
$$

If, in the formulation (2.1)-(2.6), we replace constraints (2.4) and (2.5) with the set of inequalities (3.3) for all disjoint subsets $O$ and $D$ of $I, K^{\prime}=\kappa(O, D)$, and $(j, l) \in A$, we obtain a valid formulation for HLM where we do not need to impose explicitly the integrality of $z_{j l}$ variables. For $(j, l) \in A$, constraints (2.4) linearize the nonlinear requirement

$$
z_{j l} \geq \sum_{(i, m) \in K} t_{i m} x_{i j} x_{m l}
$$

by linearizing the equivalent family of nonlinear inequalities

$$
z_{j l} \geq \sum_{(i, m) \in K^{\prime}} t_{i m} x_{i j} x_{m l}
$$

for all $K^{\prime} \subseteq K$. Inequalities (3.3) linearize the nonlinear requirement

$$
z_{j l} \geq\left\lceil\sum_{(i, m) \in K} t_{i m} x_{i j} x_{m l}\right\rceil
$$

by linearizing the equivalent family of nonlinear inequalities

$$
z_{j l} \geq\left\lceil\sum_{(i, m) \in \kappa(O, D)} t_{i m}\right\rceil \Pi_{i \in O} x_{i j} \Pi_{m \in D} x_{m l}
$$

for all disjoint subsets $O$ and $D$ of $I$.

The following example shows that it is not possible to compare the LP relaxations of these two formulations.

Example 3.1. Comparing the LP relaxation of formulation (2.1)-(2.6) with that of formulation (2.1)-(2.3), (2.6), and (3.3) is equivalent to comparing the relative strength of inequalities (2.4) and (3.3). Let $I=\{1,2,3,4\}$. Consider a vector $x$ such that $x_{12}=x_{22}=x_{34}=x_{44}=0.6$ and $x_{11}=x_{21}=x_{33}=x_{43}=0.4$ (see Figure 3.1).
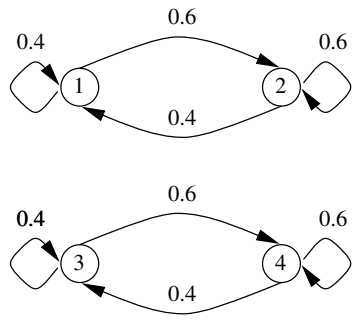

FIG. 3.1. Example 3.1: assignment of nodes.

For arc $(2,4)$ and $K^{\prime}=\{(1,3),(1,4),(2,3),(2,4)\}$, constraint $(2.4)$ reads

$$
z_{24} \geq 0.2\left(t_{13}+t_{14}+t_{23}+t_{24}\right) \text {. }
$$


This is indeed a best choice of $K^{\prime}$ in the sense that it can lead to a most violated inequality $(2.4)$ for arc $(2,4)$.

Now we consider inequality (3.3) for arc $(2,4)$. Let $O$ and $D$ be disjoint subsets of $I$. To find a most violated inequality, it is better to choose $O \subseteq\{1,2\}$ and $D \subseteq\{3,4\}$. Then, inequality $(3.3)$ is $z_{24} \geq\left\lceil\sum_{(i, m) \in \kappa(O, D)} t_{i m}\right\rceil(1-0.4|O|-0.4|D|)$. The righthand side of this inequality can be positive only if $|O|=|D|=1$. If $|O|=|D|=1$ and $i \in O$ and $m \in D$, then the inequality is

$$
z_{24} \geq 0.2\left\lceil t_{i m}\right\rceil
$$

The best inequality can be obtained by choosing a commodity $(i, m)$ with maximum $t_{i m}$. Assume without loss of generality that this maximum is attained at $i=1$ and $m=3$.

If $t_{13}+t_{14}+t_{23}+t_{24}>\left\lceil t_{13}\right\rceil$, then inequality (3.4) imposes a higher lower bound than inequality (3.5). And if $t_{13}+t_{14}+t_{23}+t_{24}<\left\lceil t_{13}\right\rceil$, then the lower bound imposed by inequality (3.5) is higher than the one imposed by inequality (3.4). Therefore, these two inequalities are not comparable.

For given sets $S$ and $T$, inequalities (3.1) can be separated in polynomial time (see [12]). However, the complexity of the separation of inequalities (3.2) is open even when $S$ and $T$ are given. Still, the separation is easy if $x$ is not fractional. In this case, sets $S$ and $T$ should be singletons and

$$
K^{\prime}=\left\{(i, m) \in K, \sum_{j \in S} x_{i j}=1 \text { and } \sum_{l \in T} x_{m l}=1\right\}
$$

Yaman and Carello [15] present inequalities that dominate the projection inequalities (3.1).

Proposition 3.5 (Yaman and Carello [15]). Let $S$ and $T$ be nonempty disjoint subsets of $I$ and $K^{\prime} \subseteq K$. The improved projection inequality

$$
\begin{aligned}
\sum_{j \in S} \sum_{l \in T} z_{j l} & \geq \sum_{(i, m) \in K^{\prime}: i \notin S, m \notin T} t_{i m}\left(\sum_{j \in S \backslash\{m\}} x_{i j}+\sum_{l \in T \backslash\{i\}} x_{m l}+x_{i m}+x_{m i}-1\right) \\
& +\sum_{(i, m) \in K^{\prime}: i \in S, m \notin T} t_{i m}\left(\sum_{j \in S \backslash\{m\}} x_{i j}+\sum_{l \in T} x_{m l}+x_{i m}-1\right) \\
& +\sum_{(i, m) \in K^{\prime}: i \notin S, m \in T} t_{i m}\left(\sum_{j \in S} x_{i j}+\sum_{l \in T \backslash\{i\}} x_{m l}+x_{m i}-1\right) \\
& +\sum_{(i, m) \in K^{\prime}: i \in S, m \in T} t_{i m}\left(\sum_{j \in S} x_{i j}+\sum_{l \in T} x_{m l}-1\right)
\end{aligned}
$$

is valid for $P$.

We present inequalities that dominate inequalities (3.2) in the same manner.

Proposition 3.6. Let $S, T, O$, and $D$ be nonempty subsets of $I$ such that 
$S \cap T=\emptyset$ and $O \cap D=\emptyset$. Inequality

$$
\begin{array}{r}
\sum_{j \in S} \sum_{l \in T} z_{j l} \geq\left\lceil\sum_{(i, m) \in \kappa(O, D)} t_{i m}\right\rceil\left[\sum_{i \in O}\left(\sum_{j \in S} x_{i j}+\sum_{m \in D \backslash(S \cup T)} x_{i m}-1\right)\right. \\
\left.+\sum_{m \in D}\left(\sum_{l \in T} x_{m l}+\sum_{i \in O \backslash(S \cup T)} x_{m i}-1\right)+1\right]
\end{array}
$$

is valid for $P$.

Proof. If $\sum_{m \in D \backslash(S \cup T)} x_{i m}=0$ for all $i \in O$ and $\sum_{i \in O \backslash(S \cup T)} x_{m i}=0$ for all $m \in D$, then inequality (3.6) reduces to inequality (3.2) for $K^{\prime}=\kappa(O, D)$.

If there exists $i \in O$ and $m \in D \backslash(S \cup T)$ such that $x_{i m}=1$ (resp., $m \in D$ and $i \in O \backslash(S \cup T)$ such that $\left.x_{m i}=1\right)$, then as $x_{m m}=1, m \notin T$ and $m \notin O$, we have $\sum_{l \in T} x_{m l}+\sum_{l \in O \backslash(S \cup T)} x_{m l}=0$ (resp., $\sum_{j \in S} x_{i j}+\sum_{j \in D \backslash(S \cup T)} x_{i j}=0$ ). This implies that the right-hand side of inequality (3.6) is nonpositive.

Inequality (3.6) remains valid if $\left\lceil\sum_{(i, m) \in \kappa(O, D)} t_{i m}\right\rceil$ is changed to $\left\lceil\sum_{(i, m) \in K^{\prime}} t_{i m}\right\rceil$ for $K^{\prime} \subset \kappa(O, D)$. But these new inequalities are dominated.

Proposition 3.7. For given nonempty subsets $S, T, O$, and $D$ of $I$ such that $S \cap T=\emptyset$ and $O \cap D=\emptyset$, inequality (3.6) dominates inequality (3.2).

Proof. If $K^{\prime}=\kappa(O, D)$, then inequality (3.6) dominates inequality (3.2). If $K^{\prime} \neq$ $\kappa(O, D)$, then by Proposition 3.4, inequality $(3.2)$ for $\kappa(O, D)$ dominates inequality (3.2) for $K^{\prime}$.

In inequality (3.2), when a node in $O\left(K^{\prime}\right)$ is assigned to some node in $I \backslash S$ or a node in $D\left(K^{\prime}\right)$ is assigned to some node in $I \backslash T$, the right-hand side of the inequality is nonpositive, since the coefficients of the assignment variables are all equal to $\left\lceil\sum_{(i, m) \in K^{\prime}} t_{i m}\right\rceil$. In the remaining part of this section, we present families of valid inequalities where the assignment variables have smaller coefficients so that even when there exist nodes in $O\left(K^{\prime}\right)$ which are assigned to nodes in $I \backslash S$ or nodes in $D\left(K^{\prime}\right)$ which are assigned to nodes in $I \backslash T$, the inequality can still give a positive lower bound on $\sum_{j \in S} \sum_{l \in T} z_{j l}$.

Proposition 3.8. Let $S$ and $T$ be nonempty disjoint subsets of $I$ and $K^{\prime} \subseteq K$. Inequality

$$
\begin{aligned}
\sum_{j \in S} \sum_{l \in T} z_{j l} \geq\left\lceil\sum_{(i, m) \in K^{\prime}} t_{i m}\right\rceil & -\sum_{i \in O\left(K^{\prime}\right)}\left(\left[\sum_{m:(i, m) \in K^{\prime}} t_{i m}\right\rceil \sum_{j \in I \backslash S} x_{i j}\right) \\
& -\sum_{m \in D\left(K^{\prime}\right)}\left(\left[\sum_{i:(i, m) \in K^{\prime}} t_{i m}\right\rceil \sum_{l \in I \backslash T} x_{m l}\right)
\end{aligned}
$$

is valid for $P$.

Proof. For a given $x$, define $O^{\prime}=\left\{i \in O\left(K^{\prime}\right): \sum_{j \in I \backslash S} x_{i j}=0\right\}$ and $D^{\prime}=\{m \in$ $\left.D\left(K^{\prime}\right): \sum_{l \in I \backslash T} x_{m l}=0\right\}$. Then the right-hand side of inequality (3.7) is equal to

$$
\begin{array}{r}
\left\lceil\sum_{(i, m) \in K^{\prime}} t_{i m}\right\rceil-\sum_{i \in O\left(K^{\prime}\right) \backslash O^{\prime}}\left\lceil\sum_{m:(i, m) \in K^{\prime}} t_{i m}\right\rceil-\sum_{m \in D\left(K^{\prime}\right) \backslash D^{\prime}}\left\lceil\sum_{i:(i, m) \in K^{\prime}} t_{i m}\right\rceil \\
\leq\left\lceil\sum_{(i, m) \in K^{\prime}: i \in O^{\prime} \text { and } m \in D^{\prime}} t_{i m}\right\rceil \leq\left\lceil\sum_{i \in O^{\prime}} \sum_{m \in D^{\prime}} t_{i m}\right\rceil .
\end{array}
$$


The last term is a valid lower bound on $\sum_{j \in S} \sum_{l \in T} z_{j l}$.

Different from inequalities (3.2) and (3.6), inequalities (3.7) defined by sets $K^{\prime} \neq$ $\kappa(O, D)$ can be nondominated. If there exists a commodity $(u, v) \in \kappa(O, D)$ such that $\left\lceil\sum_{(i, m) \in \kappa(O, D)} t_{i m}\right\rceil=\left\lceil\sum_{(i, m) \in \kappa(O, D) \backslash\{(u, v)\}} t_{i m}\right\rceil$, then inequality $(3.7)$ for $\kappa(O, D) \backslash$ $\{(u, v)\}$ either is the same as inequality $(3.7)$ for $\kappa(O, D)$ or dominates it. An example is given.

Example 3.2. Let $I=\{1,2,3,4\}$. The nonzero traffic values are as follows: $t_{13}=1.25, t_{14}=1, t_{23}=1.95, t_{24}=0.05$ (see Figure 3.2 ).

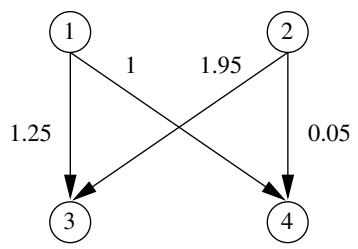

FIG. 3.2. Example 3.2: nonzero traffic values.

We consider some $\operatorname{arc}(j, l)$. Inequality $(3.7)$ for $\kappa(O, D)$, where $O=\{1,2\}$ and $D=\{3,4\}$, is

$$
\begin{aligned}
z_{j l} \geq\left\lceil t_{13}+t_{14}+t_{23}+t_{24}\right\rceil-\left\lceil t_{13}+t_{14}\right\rceil\left(1-x_{1 j}\right)-\left\lceil t_{23}+t_{24}\right\rceil\left(1-x_{2 j}\right) & \\
& -\left\lceil t_{13}+t_{23}\right\rceil\left(1-x_{3 l}\right)-\left\lceil t_{14}+t_{24}\right\rceil\left(1-x_{4 l}\right) \\
= & 5-3\left(1-x_{1 j}\right)-2\left(1-x_{2 j}\right)-4\left(1-x_{3 l}\right)-2\left(1-x_{4 l}\right) .
\end{aligned}
$$

For $K^{\prime}=\{(1,3),(1,4),(2,3)\}$, inequality $(3.7)$ is

$$
\begin{gathered}
z_{j l} \geq\left\lceil t_{13}+t_{14}+t_{23}\right\rceil-\left\lceil t_{13}+t_{14}\right\rceil\left(1-x_{1 j}\right)-\left\lceil t_{23}\right\rceil\left(1-x_{2 j}\right) \\
\quad-\left\lceil t_{13}+t_{23}\right\rceil\left(1-x_{3 l}\right)-\left\lceil t_{14}\right\rceil\left(1-x_{4 l}\right) \\
=5-3\left(1-x_{1 j}\right)-2\left(1-x_{2 j}\right)-4\left(1-x_{3 l}\right)-1\left(1-x_{4 l}\right) .
\end{gathered}
$$

Inequality (3.7) for $K^{\prime}$ dominates inequality (3.7) for $\kappa(O, D)$.

The complexity of the separation is open for inequalities (3.7). If one approximates the separation problem by removing the ceilings, then the new problem is the same as the separation problem for projection inequalities (3.1).

The coefficients of some variables can be further improved as follows.

Proposition 3.9. Let $S$ and $T$ be nonempty disjoint subsets of $I$ and $K^{\prime} \subseteq K$. For $i^{*} \in O\left(K^{\prime}\right)$, inequality

$$
\begin{aligned}
\sum_{j \in S} \sum_{l \in T} z_{j l} \geq & \left\lceil\sum_{(i, m) \in K^{\prime}} t_{i m}\right\rceil-\sum_{i \in O\left(K^{\prime}\right) \backslash i^{*}}\left(\left[\sum_{m:(i, m) \in K^{\prime}} t_{i m}\right\rceil \sum_{j \in I \backslash S} x_{i j}\right) \\
& -\left(\left\lceil\sum_{(i, m) \in K^{\prime}} t_{i m}\right\rceil-\left\lceil\sum_{(i, m) \in K^{\prime}} t_{i m}-\sum_{m:(i *, m) \in K^{\prime}} t_{i^{*} m}\right\rceil\right) \sum_{j \in I \backslash S} x_{i^{*} j} \\
& -\sum_{m \in D\left(K^{\prime}\right)}\left(\left\lceil\sum_{i:(i, m) \in K^{\prime}} t_{i m}\right\rceil \sum_{l \in I \backslash T} x_{m l}\right)
\end{aligned}
$$


is a valid inequality for $P$. Similarly, for $i^{*} \in D\left(K^{\prime}\right)$, inequality

$$
\begin{aligned}
\sum_{j \in S} \sum_{l \in T} z_{j l} \geq & \left\lceil\sum_{(i, m) \in K^{\prime}} t_{i m}\right\rceil-\sum_{i \in O\left(K^{\prime}\right)}\left(\left[\sum_{m:(i, m) \in K^{\prime}} t_{i m}\right\rceil \sum_{j \in I \backslash S} x_{i j}\right) \\
& -\left(\left\lceil\sum_{(i, m) \in K^{\prime}} t_{i m}\right\rceil-\left\lceil\sum_{(i, m) \in K^{\prime}} t_{i m}-\sum_{m:\left(m, i^{*}\right) \in K^{\prime}} t_{m i^{*}}\right]\right) \sum_{l \in I \backslash T} x_{i^{*} l} \\
& -\sum_{m \in D\left(K^{\prime}\right) \backslash i^{*}}\left(\left\lceil\sum_{i:(i, m) \in K^{\prime}} t_{i m}\right\rceil \sum_{l \in I \backslash T} x_{m l}\right)
\end{aligned}
$$

is valid for $P$.

Proof. We prove the validity of inequality (3.8). Validity of inequality (3.9) can be proved in a similar way. If $\sum_{j \in I \backslash S} x_{i^{*} j}=0$, then inequality (3.8) is the same as inequality (3.7). If $\sum_{j \in I \backslash S} x_{i^{*} j}=1$, then it is dominated by inequality (3.7) for $K^{\prime \prime}=\left\{(i, m) \in K^{\prime}: i \neq i^{*}\right\}$.

To conclude this section, we compare inequalities (3.2), (3.7), (3.8), and (3.9).

Proposition 3.10. For given nonempty disjoint subsets $S$ and $T$ of $I$ and $K^{\prime} \subseteq K$, inequality (3.7) dominates inequality (3.2) and inequalities (3.8) and (3.9) dominate inequality (3.7).

4. Facet defining inequalities. This section is devoted to the polyhedral analysis for the HLM polyhedron. We first prove some properties of the facet defining inequalities and then present families of such inequalities.

4.1. Basics. We reformulate the problem by substituting $x_{j j}=1-\sum_{m \in I \backslash\{j\}} x_{j m}$ for all $j \in I$ (see Avella and Sassano [1]). We also eliminate some inequalities (2.4). If both $j$ and $l$ become hubs, then the traffic of commodities with destination $j$ or origin $l$ does not travel on arc $(j, l)$. Moreover, the traffic from node $j$ to node $l$ travels on $\operatorname{arc}(j, l)$. Define for $(j, l) \in A$,

$$
K_{j l}=K \backslash(\{(j, l)\} \cup\{(m, j): m \in I \backslash\{j\}\} \cup\{(l, m): m \in I \backslash\{l\}\}) .
$$

The HLM can be reformulated as follows:

$$
\begin{array}{cc}
\min \sum_{i \in I} \sum_{j \in I \backslash\{i\}} C_{i j} x_{i j}+\sum_{i \in I} C_{i i}\left(1-\sum_{j \in I \backslash\{i\}} x_{i j}\right)+ & \sum_{(j, l) \in A} R_{j l} z_{j l} \\
\text { s.t. } x_{i j}+\sum_{m \in I \backslash\{j\}} x_{j m} \leq 1 & \forall(i, j) \in A, \\
z_{j l} \geq \sum_{(i, m) \in K^{\prime}, i \neq j, m \neq l} t_{i m}\left(x_{i j}+x_{m l}-1\right) & \\
& +\sum_{i \in I:(j, i) \in K^{\prime}} t_{j i}\left(x_{i l}-\sum_{m \in I \backslash\{j\}} x_{j m}\right) \\
& +\sum_{i \in I:(i, l) \in K^{\prime}} t_{i l}\left(x_{i j}-\sum_{m \in I \backslash\{l\}} x_{l m}\right)
\end{array}
$$




$$
\begin{array}{ll}
\quad+t_{j l}\left(1-\sum_{m \in I \backslash\{j\}} x_{j m}-\sum_{m \in I \backslash\{l\}} x_{l m}\right) & \forall K^{\prime} \subseteq K_{j l},(j, l) \in A, \\
x_{i j} \in\{0,1\} & \forall(i, j) \in A, \\
z_{j l} \geq 0 & \forall(j, l) \in A, \\
z_{j l} \text { integer } & \forall(j, l) \in A .
\end{array}
$$

DeFinition 4.1. Let

$$
P_{A}=\operatorname{conv}\left(\left\{(x, z) \in\{0,1\}^{n(n-1)} \times \mathbb{Z}^{n(n-1)}:(x, z) \text { satisfies }(4.1)-(4.5)\right\}\right) .
$$

Define also

$$
P_{\emptyset}=\operatorname{conv}\left(\left\{x \in\{0,1\}^{n(n-1)}: x \text { satisfies }(4.1) \text { and }(4.3)\right\}\right) .
$$

Polytope $P_{\emptyset}$ is a special stable set polytope. (See, e.g., [2], [4], and [14] for polyhedral properties of the stable set polytope and see [9] for facet defining inequalities of $P_{\emptyset}$.) Polytope $P_{\emptyset}$ is interesting since $P_{\emptyset}=\operatorname{Proj}_{x}\left(P_{A}\right)$. Labbé and Yaman [8] describe the relationship between the facets of $P_{\emptyset}$ and $P_{A}$. The following two propositions are corollaries of the results in [8] and the proofs can be found in that paper. Similar results are also proved by Labbé, Yaman, and Gourdin [12] for the polyhedron associated with HLs.

Proposition 4.2. The polyhedron $P_{A}$ is full dimensional, i.e., $\operatorname{dim}\left(P_{A}\right)=2 n(n-$ 1).

Proposition 4.3. The inequality $\pi x \leq \pi_{0}$ defines a facet of $P_{A}$ if and only if it defines a facet of $P_{\emptyset}$.

This proposition gives a characterization of the facet defining inequalities of $P_{A}$ which involve only the assignment variables, in terms of the facet defining inequalities of $P_{\emptyset}$. Next, we investigate facet defining inequalities of $P_{A}$ which involve only the capacity variables. The proofs of the following two propositions are similar to the proofs of Proposition 4.3 and 4.4 in [12] and are omitted here.

Proposition 4.4. Every facet defining inequality of $P_{A}$ of the form $\beta z \geq \beta_{0}$ is a positive multiple of $z_{j l} \geq 0$ for some $(j, l) \in A$.

This proposition implies that it is not possible to find fixed positive lower bounds on capacity variables. This is natural since if all nodes are assigned to the same hub, then there is no traffic in the backbone network.

Proposition 4.5. For $(j, l) \in A$, if $t_{j l}=0$, then the inequality $z_{j l} \geq 0$ defines a facet of $P_{A}$.

4.2. General lifting results. In what follows, we give some properties of facet defining inequalities that involve both the assignment and the capacity variables.

Define $e_{i j}^{x}=(x, z)$ (resp., $\left.e_{i j}^{z}=(x, z)\right)$ to be the unit vector such that $x_{l m}=0$ for all $(l, m) \in A \backslash\{(i, j)\}, x_{i j}=1$ and $z_{l m}=0$ for all $(l, m) \in A$ (resp., $x_{l m}=0$ for all $(l, m) \in A, z_{l m}=0$ for all $(l, m) \in A \backslash\{(i, j)\}$ and $\left.z_{i j}=1\right)$.

Definition 4.6. For $B \subseteq A$, define

$$
\begin{gathered}
F_{B}=\left\{(x, z) \in\{0,1\}^{n(n-1)} \times \mathbb{Z}^{|B|}:(x, z) \text { satisfies (4.1) and }(4.3) \forall(i, j) \in A\right. \\
\text { and (4.2), (4.4), and (4.5) } \forall(j, l) \in B\}
\end{gathered}
$$

and let

$$
P_{B}=\operatorname{conv}\left(F_{B}\right)
$$


If $B=\{(j, l)\}$, then we write $F_{j l}$ and $P_{j l}$ for $F_{B}$ and $P_{B}$, respectively.

In other words, $P_{B}$ is the projection of $P_{A}$ on the space of $x_{i j}$ for all $(i, j) \in A$ and $z_{j l}$ for all $(j, l) \in B$. Facet defining inequalities of $P_{B}$ and $P_{A}$ are related in the following way.

TheOrem 4.7. For $B \subset A$, inequality $\beta z \geq \alpha x+\pi$ with $\beta_{j l}=0$ for all $(j, l) \in$ $A \backslash B$ is facet defining for $P_{A}$ if and only if it is facet defining for $P_{B}$.

Proof. Assume that $\beta z \geq \alpha x+\pi$ with $\beta_{j l}=0$ for all $(j, l) \in A \backslash B$ is not facet defining for $P_{A}$. Then all $(x, z) \in P_{A}$ that satisfy $\beta z=\alpha x+\pi$ also satisfy $\beta^{\prime} z=\alpha^{\prime} x+\pi^{\prime}$ and $\left(\beta^{\prime}, \alpha^{\prime}, \pi^{\prime}\right) \neq 0$ is not a positive multiple of $(\beta, \alpha, \pi)$. As, for $(j, l) \in A \backslash B$, both $(x, z)$ and $(x, z)+e_{j l}^{z}$ are in $P_{A}$ and satisfy $\beta z=\alpha x+\pi$, we have $\beta_{j l}^{\prime}=0$. Then $\beta z \geq \alpha x+\pi$ cannot be facet defining for $P_{B}$.

If $\beta z \geq \alpha x+\pi$ with $\beta_{j l}=0$ for all $(j, l) \in A \backslash B$ is facet defining for $P_{A}$, then it is clearly facet defining for $P_{B}$.

Theorem 4.7 implies that for $B_{1} \subset B_{2} \subset A$, facet defining inequalities of $P_{B_{1}}$ are also facet defining for $P_{B_{2}}$. Proposition 4.3 is a special case of Theorem 4.7 where $B=\emptyset$. Facet defining inequalities of $P_{\emptyset}$ are facet defining for $P_{B}$ for every $B \subseteq A$.

Proposition 4.8. For $B \subseteq A$, if $\beta z \geq \alpha x+\pi$ is facet defining for $P_{B}$, then $\beta \geq 0$.

Proof. Let $(x, z) \in P_{B}$ be such that $\beta z=\alpha x+\pi$. As, for $(j, l) \in B,(x, z)+e_{j l}^{z}$ is also in $P_{B}, \beta_{j l} \geq 0$.

Proposition 4.8 implies that facet defining inequalities of $P_{j l}$ that involve both assignment and capacity variables are of the form $z_{j l} \geq \alpha x+\pi$. We give general properties and lifting results for these inequalities.

Definition 4.9. For $A^{\prime} \subseteq A$ and $B \subseteq A$, define

$$
F_{B}\left(A^{\prime}\right)=\left\{(x, z) \in F_{B}: x_{i m}=0 \forall(i, m) \in A \backslash A^{\prime}\right\}
$$

and

$$
P_{B}\left(A^{\prime}\right)=\operatorname{conv}\left(F_{B}\left(A^{\prime}\right)\right)
$$

If we have a facet defining inequality for $P_{B}\left(A^{\prime}\right)$, then by lifting variables $x_{i m}$ with $(i, m) \in A \backslash A^{\prime}$ sequentially, we can obtain a facet defining inequality for $P_{B}$ (see, e.g., Nemhauser and Wolsey [13]).

Proposition 4.10. For $(j, l) \in A$ and $A^{\prime} \subseteq A$, if $z_{j l} \geq \alpha x+\pi$ is facet defining for $P_{j l}\left(A^{\prime}\right)$, then $\alpha_{i m} \geq 0$ for $(i, m) \in A^{\prime}$ such that $i \neq j$ and $i \neq l$.

Proof. Let $(i, m) \in A^{\prime}$ such that $i \neq j$ and $i \neq l$. Suppose that $z_{j l} \geq \alpha x+\pi$ is facet defining for $P_{j l}\left(A^{\prime}\right)$. Then there exists $\left(x, z_{j l}\right) \in P_{j l}\left(A^{\prime}\right)$ such that $z_{j l}=\alpha x+\pi$ and $x_{i m}=1$. As $\left(x, z_{j l}\right)-e_{i m}^{x}$ is also in $P_{j l}\left(A^{\prime}\right)$, we have that $\alpha_{i m} \geq 0$.

The following three theorems give the values of the optimal lifting coefficients of some variables.

Theorem 4.11. For $(j, l) \in A, A^{\prime} \subseteq A$ and $(j, u) \in A \backslash A^{\prime}$, if inequality

$$
z_{j l} \geq \sum_{(i, m) \in A^{\prime}} \alpha_{i m} x_{i m}+\pi
$$

is facet defining for $P_{j l}\left(A^{\prime}\right)$, then

$$
z_{j l} \geq \sum_{(i, m) \in A^{\prime}} \alpha_{i m} x_{i m}+\alpha_{j u} x_{j u}+\pi
$$


is facet defining for $P_{j l}\left(A^{\prime} \cup\{(j, u)\}\right)$, where

$$
\alpha_{j u}=-\max _{x \in F_{\emptyset}\left(A^{\prime}\right)}\left(\sum_{(i, m) \in A^{\prime}: i \neq j, i \neq u \text { and } m \neq j} \alpha_{i m} x_{i m}\right)-\pi .
$$

Proof. For $x_{j u}$, the optimal lifting coefficient $\alpha_{j u}$ can be computed as

$$
\alpha_{j u}=\min _{\left(x, z_{j l}\right) \in F_{j l}\left(A^{\prime} \cup\{(j, u)\}\right): x_{j u}=1}\left(z_{j l}-\sum_{(i, m) \in A^{\prime}} \alpha_{i m} x_{i m}\right)-\pi .
$$

For a given $x$ such that $x_{j u}=1$, best choice of $z_{j l}$ is 0 . So,

$$
\alpha_{j u}=\min _{x \in F_{\emptyset}\left(A^{\prime} \cup\{(j, u)\}\right): x_{j u}=1}\left(-\sum_{(i, m) \in A^{\prime}} \alpha_{i m} x_{i m}\right)-\pi .
$$

Moreover, as $x_{j u}=1$, we have $x_{j m}=0$ for all $m \in I \backslash\{j, u\}, x_{i j}=0$ for all $i \in I \backslash\{j\}$ and $x_{u m}=0$ for all $m \in I \backslash\{u\}$.

Theorem 4.12. For $(j, l) \in A, A^{\prime} \subseteq A$ and $(l, u) \in A \backslash A^{\prime}$, if inequality (4.6) is facet defining for $P_{j l}\left(A^{\prime}\right)$, then

$$
z_{j l} \geq \sum_{(i, m) \in A^{\prime}} \alpha_{i m} x_{i m}+\alpha_{l u} x_{l u}+\pi
$$

is facet defining for $P_{j l}\left(A^{\prime} \cup\{(l, u)\}\right)$, where

$$
\alpha_{l u}=-\max _{x \in F_{\emptyset}\left(A^{\prime}\right)}\left(\sum_{(i, m) \in A^{\prime}: i \neq l, i \neq u \text { and } m \neq l} \alpha_{i m} x_{i m}\right)-\pi .
$$

Proof. The proof is analogous to the proof of Theorem 4.11.

Theorem 4.13. For $(j, l) \in A$ and $A^{\prime} \subset A$, assume that inequality (4.6) is facet defining for $P_{j l}\left(A^{\prime}\right)$. Let $(u, v) \in A \backslash A^{\prime}$ such that $u$ is different from $j$ and $l$. Consider the two sets of conditions (i) and (ii):

(i) (a) $(j, v) \in A^{\prime}$,

(b) for each $m \in I \backslash\{u, v, j\}$ independently, we have $(u, m) \in A \backslash A^{\prime}$ or $\alpha_{u m}=0$,

(c) for each $m \in I \backslash\{u, v, j\}$ independently, we have $(m, u) \in A \backslash A^{\prime}$ or $\alpha_{m u}=0$.

(ii) (a) $(l, v) \in A^{\prime}$,

(b) for each $m \in I \backslash\{u, v, l\}$ independently, we have $(u, m) \in A \backslash A^{\prime}$ or $\alpha_{u m}=0$,

(c) for each $m \in I \backslash\{u, v, l\}$ independently, we have $(m, u) \in A \backslash A^{\prime}$ or $\alpha_{m u}=0$.

If at least one set of conditions (i) and (ii) is satisfied, then inequality (4.6) is also facet defining for $P_{j l}\left(A^{\prime} \cup\{(u, v)\}\right)$.

Proof. If inequality (4.6) is facet defining for $P_{j l}\left(A^{\prime}\right)$, then inequality

$$
z_{j l} \geq \sum_{(i, m) \in A^{\prime}} \alpha_{i m} x_{i m}+\alpha_{u v} x_{u v}+\pi
$$


is facet defining for $P_{j l}\left(A^{\prime} \cup\{(u, v)\}\right)$, where

$$
\alpha_{u v}=\min _{\left(x, z_{j l}\right) \in P_{j l}\left(A^{\prime} \cup\{(u, v)\}\right): x_{u v}=1}\left(z_{j l}-\sum_{(i, m) \in A^{\prime}} \alpha_{i m} x_{i m}\right)-\pi .
$$

Assume that condition set (i) is satisfied. As inequality (4.6) is facet defining for $P_{j l}\left(A^{\prime}\right)$ and by condition set (i), we know that there exists $\left(x, z_{j l}\right)$ in $P_{j l}\left(A^{\prime}\right)$ such that $x_{j v}=1, z_{j l}=\sum_{(i, m) \in A^{\prime}} \alpha_{i m} x_{i m}+\pi$ and $\sum_{m \in I \backslash\{u, v, j\}}\left(x_{u m}+x_{m u}\right)=0$. Then $\left(x, z_{j l}\right)+e_{u v}^{x}$ is in $P_{j l}\left(A^{\prime} \cup\{(u, v)\}\right)$ and so $\alpha_{u v} \leq 0$. By Proposition 4.10, $\alpha_{u v} \geq 0$. Thus $\alpha_{u v}=0$. The case where condition set (ii) is satisfied is similar.

We conclude this section with two more lifting theorems.

Let $(j, l) \in A, I_{j} \subseteq I \backslash\{j, l\}, I_{l} \subseteq I \backslash\{j, l\}$ and $A^{\prime}=\left\{(i, j): i \in I_{j}\right\} \cup\{(m, l)$ : $\left.m \in I_{l}\right\}$. Consider inequality

$$
z_{j l} \geq \sum_{i \in I_{j}} \alpha_{i j} x_{i j}+\sum_{m \in I_{l}} \alpha_{m l} x_{m l}+\pi
$$

which is facet defining for $P_{j l}\left(A^{\prime}\right)$. Let $u \in I \backslash\left(I_{j} \cup\{j, l\}\right)$. To compute the lifting coefficient of the variable $x_{u j}$, we solve a min cut problem on a directed layer graph $G_{u j}=\left(N_{u j}, A_{u j}\right)$ constructed as follows. Let $I_{j}^{\prime}=\left\{i \in I_{j}: \alpha_{i j}-t_{i l}>0\right\}$ and $I_{l}^{\prime}=\left\{m \in I_{l}: \alpha_{m l}-t_{j m}-t_{u m}>0\right\}$. Let $o$ and $d$ be two dummy nodes. The node set is $N_{u j}=\{o, d\} \cup I_{j}^{\prime} \cup I_{l}^{\prime}$. The first layer includes node $o$, the second layer includes nodes of $I_{j}^{\prime}$, the third layer includes nodes of $I_{l}^{\prime}$, and the fourth layer includes node $d$. Arcs go from the nodes of a layer to the nodes of the next layer. Thus, the arc set consists of arcs from node $o$ to nodes in $I_{j}^{\prime}$, arcs from nodes in $I_{j}^{\prime}$ to nodes in $I_{l}^{\prime}$, and arcs from nodes in $I_{l}^{\prime}$ to node $d$, i.e., $A_{u j}=\left\{(o, i): i \in I_{j}^{\prime}\right\} \cup\left\{(i, m): i \in I_{j}^{\prime}, m \in\right.$ $\left.I_{l}^{\prime}\right\} \cup\left\{(m, d): m \in I_{l}^{\prime}\right\}$. A cut separating nodes $o$ and $d$ is defined by a subset $C \subset N_{u j}$ with $o \in C$ and $d \notin C$, and the capacity of the cut is the sum of the capacities of arcs going from nodes of $C$ to nodes of $N_{u j} \backslash C$. If there is no such arc, then the cut has zero capacity.

Theorem 4.14. Let $(j, l) \in A, I_{j} \subseteq I \backslash\{j, l\}, I_{l} \subseteq I \backslash\{j, l\}$, and $A^{\prime}=\{(i, j):$ $\left.i \in I_{j}\right\} \cup\left\{(m, l): m \in I_{l}\right\}$. Consider inequality (4.7) with integer coefficients.

Let $u \in I \backslash\left(I_{j} \cup\{j, l\}\right)$ and define $I_{j}^{\prime}=\left\{i \in I_{j}: \alpha_{i j}-t_{i l}>0\right\}$ and $I_{l}^{\prime}=\left\{m \in I_{l}\right.$ : $\left.\alpha_{m l}-t_{j m}-t_{u m}>0\right\}$. Consider the graph $G_{u j}=\left(N_{u j}, A_{u j}\right)$ constructed above. The capacity of arc $(i, m) \in A_{u j}$ is as follows:

$$
w_{i m}= \begin{cases}\alpha_{m j}-t_{m l} & \text { if } i=o \text { and } m \in I_{j}^{\prime}, \\ \infty & \text { if } i=m \text { and } i \in I_{j}^{\prime} \cap I_{l}^{\prime}, \\ t_{i m} & \text { if } i \in I_{j}^{\prime} \text { and } m \in I_{l}^{\prime} \backslash\{i\}, \\ \alpha_{i l}-t_{j i}-t_{u i} & \text { if } m=d \text { and } i \in I_{l}^{\prime} .\end{cases}
$$

Let $\omega$ be the capacity of a minimum capacity cut separating nodes o and $d$ in the graph $G_{u j}=\left(N_{u j}, A_{u j}\right)$. Compute

$$
\alpha_{u j}=-\pi+\left\lceil t_{j l}+t_{u l}-\sum_{i \in I_{j}^{\prime}}\left(\alpha_{i j}-t_{i l}\right)-\sum_{m \in I_{l}^{\prime}}\left(\alpha_{m l}-t_{j m}-t_{u m}\right)+\omega\right\rceil .
$$


If inequality (4.7) is facet defining for $P_{j l}\left(A^{\prime}\right)$, then inequality

$$
z_{j l} \geq \sum_{i \in I_{j}} \alpha_{i j} x_{i j}+\sum_{m \in I_{l}} \alpha_{m l} x_{m l}+\alpha_{u j} x_{u j}+\pi
$$

is facet defining for $P_{j l}\left(A^{\prime} \cup\{(u, j)\}\right)$.

Proof. The optimal lifting coefficient of $x_{u j}$ can be computed as follows:

$$
\begin{array}{r}
\alpha_{u j}=-\pi+\min _{\left(x, z_{j l}\right) \in F_{j l}\left(A^{\prime} \cup\{(u, j)\}\right): x_{u j}=1}\left(z_{j l}-\sum_{i \in I_{j}} \alpha_{i j} x_{i j}-\sum_{m \in I_{l}} \alpha_{m l} x_{m l}\right) \\
=-\pi+\min _{\left(x, z_{j l}\right) \in F_{j l}\left(A^{\prime} \cup\{(u, j)\}\right): x_{u j}=1}\left\lceil t_{j l}+t_{u l}+\sum_{i \in I_{j}}\left(t_{i l}-\alpha_{i j}\right) x_{i j}\right. \\
\left.+\sum_{m \in I_{l}}\left(t_{j m}+t_{u m}-\alpha_{m l}\right) x_{m l}+\sum_{i \in I_{j}} \sum_{m \in I_{l}} t_{i m} x_{i j} x_{m l}\right] .
\end{array}
$$

There is an optimal solution where $x_{i j}=0$ for all $i \in I_{j} \backslash I_{j}^{\prime}$ and $x_{m l}=0$ for all $m \in I_{l} \backslash I_{l}^{\prime}$. So,

$$
\begin{aligned}
\alpha_{u j}=-\pi+\left\lceil t_{j l}+\right. & t_{u l}+\min _{\left(x, z_{j l}\right) \in F_{j l}\left(A^{\prime} \cup\{(u, j)\}\right): x_{u j}=1}\left(\sum_{i \in I_{j}^{\prime}}\left(t_{i l}-\alpha_{i j}\right) x_{i j}\right. \\
& \left.\left.+\sum_{m \in I_{l}^{\prime}}\left(t_{j m}+t_{u m}-\alpha_{m l}\right) x_{m l}+\sum_{i \in I_{j}^{\prime}} \sum_{m \in I_{l}^{\prime}} t_{i m} x_{i j} x_{m l}\right)\right] \\
=-\pi+\left[t_{j l}+\right. & t_{u l}-\sum_{i \in I_{j}^{\prime}}\left(\alpha_{i j}-t_{i l}\right)-\sum_{m \in I_{l}^{\prime}}\left(\alpha_{m l}-t_{j m}-t_{u m}\right) \\
& +\min _{\left(x, z_{j l}\right) \in F_{j l}\left(A^{\prime} \cup\{(u, j)\}\right): x_{u j}=1}\left(\sum_{i \in I_{j}^{\prime}}\left(\alpha_{i j}-t_{i l}\right)\left(1-x_{i j}\right)\right. \\
& \left.\left.+\sum_{m \in I_{l}^{\prime}}\left(\alpha_{m l}-t_{j m}-t_{u m}\right)\left(1-x_{m l}\right)+\sum_{i \in I_{j}^{\prime}} \sum_{m \in I_{l}^{\prime}} t_{i m} x_{i j} x_{m l}\right)\right] .
\end{aligned}
$$

It remains to show that $\omega$ is equal to the optimal value of the above minimization problem. Let $C$ be a cut separating nodes $o$ and $d$ in $G_{u j}$. The capacity of cut $C$ is

$$
\sum_{i \in I_{j}^{\prime} \backslash C}\left(\alpha_{i j}-t_{i l}\right)+\sum_{m \in I_{l}^{\prime} \cap C}\left(\alpha_{m l}-t_{j m}-t_{u m}\right)+\sum_{i \in I_{j}^{\prime} \cap C} \sum_{m \in I_{l}^{\prime} \backslash C} t_{i m} .
$$

This is the cost of a solution where $x_{i j}$ is equal to 1 if $i \in I_{j}^{\prime} \cap C$ and 0 otherwise for $i \in I_{j}^{\prime}$ and $x_{m l}$ is equal to 1 if $m \in I_{l}^{\prime} \backslash C$ and 0 otherwise for $m \in I_{l}^{\prime}$. The solution is infeasible if there exists $i \in I_{j}^{\prime} \cap I_{l}^{\prime}$ such that $x_{i j}+x_{i l}=2$. Then the corresponding cut has infinite capacity since $w_{i i}=\infty$ for all $i \in I_{j}^{\prime} \cap I_{l}^{\prime}$. Therefore, any feasible solution of the minimization problem is a cut with a finite capacity and vice versa. Besides, the cost of a feasible solution is the same as the capacity of the corresponding cut. So $\omega$ is the same as the optimal value of the minimization problem. 
Theorem 4.15. Let $(j, l) \in A, I_{j} \subseteq I \backslash\{j, l\}, I_{l} \subseteq I \backslash\{j, l\}$, and $A^{\prime}=\{(i, j)$ : $\left.i \in I_{j}\right\} \cup\left\{(m, l): m \in I_{l}\right\}$. Consider inequality (4.7) with integer coefficients.

Let $u \in I \backslash\left(I_{l} \cup\{j, l\}\right)$ and define $I_{j}^{\prime}=\left\{i \in I_{j}: \alpha_{i j}-t_{i l}-t_{i u}>0\right\}$ and $I_{l}^{\prime}=\left\{m \in I_{l}: \alpha_{m l}-t_{j m}>0\right\}$. Consider the graph $G_{u l}=\left(N_{u l}, A_{u l}\right)$. The node set is $N_{u l}=\{o, d\} \cup I_{j}^{\prime} \cup I_{l}^{\prime}$, and nodes $o$ and $d$ are dummy nodes. The arc set is $A_{u l}=\left\{(o, i): i \in I_{j}^{\prime}\right\} \cup\left\{(i, m): i \in I_{j}^{\prime}, m \in I_{l}^{\prime}\right\} \cup\left\{(m, d): m \in I_{l}^{\prime}\right\}$. The capacity of $\operatorname{arc}(i, m) \in A_{u l}$ is as follows:

$$
w_{i m}= \begin{cases}\alpha_{m j}-t_{m l}-t_{m u} & \text { if } i=o \text { and } m \in I_{j}^{\prime}, \\ \infty & \text { if } i=m \text { and } i \in I_{j}^{\prime} \cap I_{l}^{\prime}, \\ t_{i m} & \text { if } i \in I_{j}^{\prime} \text { and } m \in I_{l}^{\prime} \backslash\{i\}, \\ \alpha_{i l}-t_{j i} & \text { if } m=d \text { and } i \in I_{l}^{\prime} .\end{cases}
$$

Let $\omega$ be the capacity of a minimum capacity cut separating nodes o and $d$ in the graph $G_{u l}=\left(N_{u l}, A_{u l}\right)$. Compute

$$
\alpha_{u l}=-\pi+\left\lceil t_{j l}+t_{j u}-\sum_{i \in I_{j}^{\prime}}\left(\alpha_{i j}-t_{i l}-t_{i u}\right)-\sum_{m \in I_{l}^{\prime}}\left(\alpha_{m l}-t_{j m}\right)+\omega\right\rceil .
$$

If inequality (4.7) is facet defining for $P_{j l}\left(A^{\prime}\right)$, then inequality

$$
z_{j l} \geq \sum_{i \in I_{j}} \alpha_{i j} x_{i j}+\sum_{m \in I_{l}} \alpha_{m l} x_{m l}+\alpha_{u l} x_{u l}+\pi
$$

is facet defining for $P_{j l}\left(A^{\prime} \cup\{(u, l)\}\right)$.

4.3. Facets of $\boldsymbol{P}_{\boldsymbol{j} \boldsymbol{l}}$. We present families of facet defining inequalities of $P_{j l}$ for $(j, l) \in A$. By Theorem 4.7, these inequalities are also facet defining for $P_{A}$.

We use sequential lifting to derive facet defining inequalities for $P_{j l}$. We start with the inequality $z_{j l} \geq\left\lceil t_{j l}\right\rceil$, which is facet defining for $P_{j l}(\emptyset)$. For a subset $I^{\prime} \subseteq I \backslash\{j, l\}$ and an order $\phi$ on $I^{\prime}$, we first lift the variables $x_{i j}$ for $i \in I^{\prime}$ in the order $\phi$. The remaining variables are lifted in the following order: $x_{j m}$ for $m \in I \backslash\{j\}, x_{u v}$ with $u \in I \backslash\{j, l\}$ and $v \in I \backslash\{j, u\}, x_{l m}$ with $m \in I \backslash\{l\}$, and $x_{u j}$ with $u \in I \backslash\left(I^{\prime} \cup\{j, l\}\right)$. As all lifting coefficients are optimal, the resulting inequality is facet defining for $P_{j l}$.

Theorem 4.16. Let $(j, l) \in A, I^{\prime} \subseteq I \backslash\{j, l\}$ and $\phi$ be an order on $I^{\prime}$. For $i \in I^{\prime}$,

$$
\alpha_{i j}=-\left\lceil t_{j l}\right\rceil+\left\lceil t_{j l}+t_{i l}-\sum_{m \in I^{\prime}: \phi(m)<\phi(i)}\left(\alpha_{m j}-t_{m l}\right)^{+}\right\rceil .
$$

Inequality

(4.8) $z_{j l} \geq\left\lceil t_{j l}\right\rceil\left(1-\sum_{m \in I \backslash\{j\}} x_{j m}-\sum_{m \in I \backslash\{l\}} x_{l m}\right)+\sum_{i \in I^{\prime}} \alpha_{i j}\left(x_{i j}-\sum_{m \in I \backslash\{l, i\}} x_{l m}\right)$

is facet defining for $P_{j l}$.

Proof. Inequality $z_{j l} \geq\left\lceil t_{j l}\right\rceil$ is facet defining for $P_{j l}(\emptyset)$. We lift variables $x_{i j}$ for $i \in I^{\prime}$ in the order $\phi$. Let

$$
F_{j l}^{i}=\left\{\left(x, z_{j l}\right) \in F_{j l}(\{(m, j) \in A: \phi(m) \leq \phi(i)\}): x_{i j}=1\right\} .
$$


The optimal lifting coefficient for $x_{i j}$ is

$$
\alpha_{i j}=\min _{\left(x, z_{j l}\right) \in F_{j l}^{i}}\left(z_{j l}-\left\lceil t_{j l}\right\rceil-\sum_{m \in I^{\prime}: \phi(m)<\phi(i)} \alpha_{m j} x_{m j}\right) .
$$

For $x$ such that $x_{i j}=1$, the lowest value of $z_{j l}$ is

$$
\left\lceil t_{j l}+t_{i l}+\sum_{m \in I^{\prime}: \phi(m)<\phi(i)} t_{m l} x_{m j}\right\rceil .
$$

Thus,

$\alpha_{i j}=\min _{\left(x, z_{j l}\right) \in F_{j l}^{i}}\left(\left\lceil t_{j l}+t_{i l}+\sum_{m \in I^{\prime}: \phi(m)<\phi(i)} t_{m l} x_{m j}\right\rceil-\sum_{m \in I^{\prime}: \phi(m)<\phi(i)} \alpha_{m j} x_{m j}\right)-\left\lceil t_{j l}\right\rceil$.

By induction, one can show that $\alpha_{m j}$ is an integer for each $m \in I^{\prime}$ such that $\phi(m)<$ $\phi(i)$. So,

$$
\alpha_{i j}=-\left\lceil t_{j l}\right\rceil+\min _{\left(x, z_{j l}\right) \in F_{j l}^{i}}\left\lceil t_{j l}+t_{i l}+\sum_{m \in I^{\prime}: \phi(m)<\phi(i)}\left(t_{m l}-\alpha_{m j}\right) x_{m j}\right\rceil .
$$

The minimization problem can be solved by setting $x_{m j}=1$ for $m \in I^{\prime}$ with $\phi(m)<$ $\phi(i)$ if $\alpha_{m j}-t_{m l} \geq 0$ and at 0 otherwise.

Next we lift variables $x_{j m}$. For $m \in I \backslash\{j\}$, Theorem 4.11 implies that $\alpha_{j m}=$ $-\left\lceil t_{j l}\right\rceil$.

Now consider some $x_{u v}$ with $u \in I \backslash\{j, l\}$ and $v \in I \backslash\{j, u\}$. We prove by induction that $\alpha_{u v}=0$. If $x_{u v}$ is the first variable with $u \in I \backslash\{j, l\}$ and $v \in I \backslash\{j, u\}$ to lift, then as $x_{j v}$ is already lifted and for each $m \in I \backslash\{u, v, j\}, x_{u m}$ and $x_{m u}$ are not yet lifted, condition set (i) of Theorem 4.13 is satisfied and the lifting coefficient of $x_{u v}$ is zero. Otherwise, assume that those $x_{i m}$ with $i \in I \backslash\{j, l\}$ and $m \in I \backslash\{j, i\}$ that are already lifted have zero coefficient. Then as $x_{j v}$ is already lifted and for each $m \in I \backslash\{u, v, j\}, x_{u m}$ is not lifted or it has zero lifting coefficient and $x_{m u}$ is not lifted or it has zero lifting coefficient, condition set (i) of Theorem 4.13 is satisfied. Hence, the lifting coefficient of $x_{u v}$ is zero.

We lift variables $x_{l m}$. For $m \in I \backslash\{l\}$, as by Proposition $4.10 \alpha_{i j} \geq 0$ for all $i \in I^{\prime}$ and $\alpha_{j i} \leq 0$ for all $i \in I \backslash\{j\}$, Theorem 4.12 implies that $\alpha_{l m}=-\sum_{i \in I^{\prime} \backslash\{m\}} \alpha_{i j}-\left\lceil t_{j l}\right\rceil$.

Finally variables $x_{u j}$ with $u \in I \backslash\left(I^{\prime} \cup\{j, l\}\right)$ are lifted by applying Theorem 4.13 repeatedly. As $x_{l j}$ is already lifted and for each $m \in I \backslash\{u, j, l\}$, the lifting coefficients of $x_{u m}$ and $x_{m u}$ are zero, condition set (ii) of Theorem 4.13 is satisfied and the lifting coefficient of $x_{u j}$ is zero.

The three corollaries below present facet defining inequalities that are special cases of inequalities (4.8) for $\left|I^{\prime}\right| \leq 2$.

Corollary 4.17. For $(j, l) \in A$, inequality

$$
z_{j l} \geq\left\lceil t_{j l}\right\rceil\left(1-\sum_{m \in I \backslash\{j\}} x_{j m}-\sum_{m \in I \backslash\{l\}} x_{l m}\right)
$$

is facet defining for $P_{j l}$. 
Corollary 4.18. For $(j, l) \in A$ and $u \in I \backslash\{j, l\}$, inequality

$$
\begin{aligned}
z_{j l} \geq & \left\lceil t_{j l}\right\rceil\left(1-\sum_{m \in I \backslash\{j\}} x_{j m}-\sum_{m \in I \backslash\{l\}} x_{l m}\right) \\
& +\left(\left\lceil t_{j l}+t_{u l}\right\rceil-\left\lceil t_{j l}\right\rceil\right)\left(x_{u j}-\sum_{m \in I \backslash\{l, u\}} x_{l m}\right)
\end{aligned}
$$

is facet defining for $P_{j l}$.

Corollary 4.19. Let $(j, l) \in A$ and $u, v \in I \backslash\{j, l\}$ such that $u \neq v$. Let $a=\min \left\{\left\lceil t_{j l}+t_{u l}+t_{v l}\right\rceil-\left\lceil t_{j l}+t_{u l}\right\rceil,\left\lceil t_{j l}+t_{v l}\right\rceil-\left\lceil t_{j l}\right\rceil\right\}$. Inequality

$$
\begin{aligned}
z_{j l} \geq & \left\lceil t_{j l}\right\rceil\left(1-\sum_{m \in I \backslash\{j\}} x_{j m}-\sum_{m \in I \backslash\{l\}} x_{l m}\right)+a\left(x_{v j}-\sum_{m \in I \backslash\{l, v\}} x_{l m}\right) \\
& +\left(\left\lceil t_{j l}+t_{u l}\right\rceil-\left\lceil t_{j l}\right\rceil\right)\left(x_{u j}-\sum_{m \in I \backslash\{l, u\}} x_{l m}\right)
\end{aligned}
$$

is facet defining for $P_{j l}$.

Theorem 4.20. Let $(j, l) \in A, I^{\prime} \subseteq I \backslash\{j, l\}$ and $\phi$ be an order on $I^{\prime}$. For $i \in I^{\prime}$,

$$
\alpha_{i l}=-\left\lceil t_{j l}\right\rceil+\left\lceil t_{j l}+t_{j i}-\sum_{m \in I^{\prime}: \phi(m)<\phi(i)}\left(\alpha_{m l}-t_{j m}\right)^{+}\right\rceil .
$$

Inequality

$$
z_{j l} \geq\left\lceil t_{j l}\right\rceil\left(1-\sum_{m \in I \backslash\{j\}} x_{j m}-\sum_{m \in I \backslash\{l\}} x_{l m}\right)+\sum_{i \in I^{\prime}} \alpha_{i l}\left(x_{i l}-\sum_{m \in I \backslash\{j, i\}} x_{j m}\right)
$$

is facet defining for $P_{j l}$.

Proof. Analogous to the proof of Theorem 4.16.

Facet defining inequalities can also be obtained by fixing the values of some variables to 1 and applying sequential lifting.

Let $A_{0}$ and $A_{1}$ be disjoint subsets of $A$. For $(j, l) \in A$, define

$$
\bar{F}_{j l}\left(A_{0}, A_{1}\right)=F_{j l} \cap\left\{\left(x, z_{j l}\right): x_{i m}=0 \forall(i, m) \in A_{0} \text { and } x_{i m}=1 \forall(i, m) \in A_{1}\right\}
$$

and

$$
\bar{P}_{j l}\left(A_{0}, A_{1}\right)=\operatorname{conv}\left(\bar{F}_{j l}\left(A_{0}, A_{1}\right)\right) .
$$

Let $I^{\prime} \subseteq I \backslash\{j, l\}$ and $A_{1}=\left\{(i, j) \in A: i \in I^{\prime}\right\}$. Inequality $z_{j l} \geq\left\lceil\sum_{m \in I^{\prime}} t_{m l}+t_{j l}\right\rceil$ is facet defining for $\bar{P}_{j l}\left(A \backslash A_{1}, A_{1}\right)$. To derive a facet defining inequality for $P_{j l}$, we first lift $\left(1-x_{i j}\right)$ for $i \in I^{\prime}$ in some order $\phi$, then $x_{j m}$ for $m \in I \backslash\{j\}, x_{u v}$ with $u \in I \backslash\{j, l\}$ and $v \in I \backslash\{j, u\}, x_{l m}$ with $m \in I \backslash\{l\}$, and finally $x_{u j}$ with $u \in I \backslash\left(I^{\prime} \cup\{j, l\}\right)$.

Theorem 4.21. Let $(j, l) \in A, I^{\prime} \subseteq I \backslash\{j, l\}$ and $\phi$ be an order on $I^{\prime}$. For $i \in I^{\prime}$,

$$
\alpha_{i j}=-\left\lceil\sum_{m \in I^{\prime}} t_{m l}+t_{j l}\right\rceil+\left\lceil t_{j l}+\sum_{m \in I^{\prime} \backslash\{i\}} t_{m l}-\sum_{m \in I^{\prime}: \phi(m)<\phi(i)}\left(t_{m l}+\alpha_{m j}\right)^{+}\right\rceil .
$$


Inequality

$$
\begin{aligned}
z_{j l} \geq \sum_{i \in I^{\prime}} \alpha_{i j}(1- & \left.x_{i j}-x_{l i}-\sum_{m \in I \backslash\{j\}} x_{j m}\right) \\
+ & {\left[\sum_{m \in I^{\prime}} t_{m l}+t_{j l}\right]\left(1-\sum_{m \in I \backslash\{j\}} x_{j m}-\sum_{m \in I \backslash\{l\}} x_{l m}\right) }
\end{aligned}
$$

is facet defining for $P_{j l}$.

Proof. Let $A_{1}=\left\{(i, j) \in A: i \in I^{\prime}\right\}$. Inequality $z_{j l} \geq\left\lceil\sum_{m \in I^{\prime}} t_{m l}+t_{j l}\right\rceil$ is facet defining for $\bar{P}_{j l}\left(A \backslash A_{1}, A_{1}\right)$. We lift $\left(1-x_{i j}\right)$ for $i \in I^{\prime}$ in the order $\phi$. Let

$$
F_{j l}^{i}=\bar{F}_{j l}\left(A \backslash A_{1} \cup\{(i, j)\}, A_{1} \backslash\{(m, j): \phi(m) \leq \phi(i)\}\right) .
$$

The optimal lifting coefficient for $\left(1-x_{i j}\right)$ is

$$
\alpha_{i j}=\min _{\left(x, z_{j l}\right) \in F_{j l}^{i}}\left(z_{j l}-\sum_{m \in I^{\prime}: \phi(m)<\phi(i)} \alpha_{m j}\left(1-x_{m j}\right)\right)-\left\lceil\sum_{m \in I^{\prime}} t_{m l}+t_{j l}\right\rceil .
$$

For $x$ such that $x_{i j}=0$, the lowest value for $z_{j l}$ is

$$
z_{j l}=\left\lceil t_{j l}+\sum_{m \in I^{\prime}: \phi(m)>\phi(i)} t_{m l}+\sum_{m \in I^{\prime}: \phi(m)<\phi(i)} t_{m l} x_{m j}\right\rceil .
$$

Then

$$
\begin{aligned}
\alpha_{i j}=\min _{\left(x, z_{j l}\right) \in F_{j l}^{i}} & \left(\left\lceil t_{j l}+\sum_{m \in I^{\prime}: \phi(m)>\phi(i)} t_{m l}+\sum_{m \in I^{\prime}: \phi(m)<\phi(i)} t_{m l} x_{m j}\right\rceil\right. \\
& \left.-\sum_{m \in I^{\prime}: \phi(m)<\phi(i)} \alpha_{m j}\left(1-x_{m j}\right)\right)-\left\lceil\sum_{m \in I^{\prime}} t_{m l}+t_{j l}\right\rceil .
\end{aligned}
$$

By induction, one can again show that $\alpha_{m j}$ is integer for each $m \in I^{\prime}$ such that $\phi(m)<\phi(i)$. So

$$
\begin{aligned}
\alpha_{i j} & =-\left\lceil\sum_{m \in I^{\prime}} t_{m l}+t_{j l}\right]+\min _{\left(x, z_{j l}\right) \in F_{j l}^{i}}\left\lceil t_{j l}+\sum_{m \in I^{\prime} \backslash\{i\}} t_{m l}\right. \\
& \left.-\sum_{m \in I^{\prime}: \phi(m)<\phi(i)}\left(t_{m l}+\alpha_{m j}\right)\left(1-x_{m j}\right)\right] \\
& =-\left[\sum_{m \in I^{\prime}} t_{m l}+t_{j l}\right]+\left\lceil t_{j l}+\sum_{m \in I^{\prime} \backslash\{i\}} t_{m l}-\sum_{m \in I^{\prime}: \phi(m)<\phi(i)}\left(t_{m l}+\alpha_{m j}\right)^{+}\right] .
\end{aligned}
$$

Next, we lift variables $x_{j m}$. For $m \in I \backslash\{j\}, \alpha_{j m}=-\sum_{i \in I^{\prime}} \alpha_{i j}-\left\lceil\sum_{i \in I^{\prime}} t_{i l}+t_{j l}\right\rceil$ since $x_{i j}=0$ for all $i \in I^{\prime}$ as $x_{j m}=1$.

Now we lift variables $x_{u v}$ with $u \in I \backslash\{j, l\}$ and $v \in I \backslash\{j, u\}$. As condition set (i) of Theorem 4.13 is satisfied, these variables have zero lifting coefficient (see the proof of Theorem 4.16). 
Next, we lift variables $x_{l m}$. Let $m \in I \backslash\{j, l\}$. Since $\alpha_{i j} \leq 0$ for all $i \in I^{\prime}$ and $\alpha_{j i}$ is the same for all $i \in I \backslash\{j\}$, by Theorem 4.12, the optimal lifting coefficient for $x_{l m}$ is

$$
\alpha_{l m}=-\sum_{i \in I^{\prime}} \alpha_{i j}-\left\lceil\sum_{i \in I^{\prime}} t_{i l}+t_{j l}\right\rceil+\min \left\{\sum_{i \in I^{\prime} \backslash\{m\}} \alpha_{i j}, \sum_{i \in I^{\prime}} \alpha_{i j}+\left\lceil\sum_{i \in I^{\prime}} t_{i l}+t_{j l}\right\rceil\right\} .
$$

If $m \notin I^{\prime}$, then $\alpha_{l m}=-\left\lceil\sum_{i \in I^{\prime}} t_{i l}+t_{j l}\right\rceil$. If $m \in I^{\prime}$, then

$$
\alpha_{l m}=-\sum_{i \in I^{\prime}} \alpha_{i j}-\left\lceil\sum_{i \in I^{\prime}} t_{i l}+t_{j l}\right\rceil+\min \left\{\sum_{i \in I^{\prime}} \alpha_{i j}-\alpha_{m j}, \sum_{i \in I^{\prime}} \alpha_{i j}+\left\lceil\sum_{i \in I^{\prime}} t_{i l}+t_{j l}\right\rceil\right\} .
$$

This is the same as $\min \left\{-\alpha_{m j}-\left\lceil\sum_{i \in I^{\prime}} t_{i l}+t_{j l}\right\rceil, 0\right\}$. As

$$
\left\lceil t_{j l}+\sum_{i \in I^{\prime} \backslash\{m\}} t_{i l}-\sum_{i \in I^{\prime}: \phi(i)<\phi(m)}\left(t_{i l}+\alpha_{i j}\right)^{+}\right\rceil \geq 0
$$

we get $\alpha_{l m}=-\alpha_{m j}-\left\lceil\sum_{i \in I^{\prime}} t_{i l}+t_{j l}\right\rceil$.

We lift $x_{l j}$. As $\sum_{i \in I \backslash\{j\}} x_{j i}=0, \alpha_{l j}=-\left\lceil\sum_{i \in I^{\prime}} t_{i l}+t_{j l}\right\rceil$.

Finally variables $x_{u j}$ with $u \in I \backslash\left(I^{\prime} \cup\{j, l\}\right)$ are lifted by applying Theorem 4.13 repeatedly and their lifting coefficients are zero (see proof of Theorem 4.16).

The resulting inequality is

$$
\begin{aligned}
z_{j l} \geq & \left\lceil\sum_{m \in I^{\prime}} t_{m l}+t_{j l}\right\rceil+\sum_{i \in I^{\prime}} \alpha_{i j}\left(1-x_{i j}\right)-\sum_{m \in I \backslash\{j\}}\left(\sum_{i \in I^{\prime}} \alpha_{i j}+\left\lceil\sum_{i \in I^{\prime}} t_{i l}+t_{j l}\right\rceil\right) x_{j m} \\
& -\sum_{m \in I \backslash\left(I^{\prime} \cup\{l\}\right)}\left\lceil\sum_{i \in I^{\prime}} t_{i l}+t_{j l}\right\rceil x_{l m}-\sum_{m \in I^{\prime}}\left(\alpha_{m j}+\left\lceil\sum_{i \in I^{\prime}} t_{i l}+t_{j l}\right\rceil\right) x_{l m} .
\end{aligned}
$$

Rearranging terms, we obtain inequality (4.13).

For $I^{\prime}=\emptyset$ and $I^{\prime}=\{u\}$, inequality (4.13) reduces to inequalities (4.9) and (4.10), respectively. Inequality (4.13) for $I^{\prime}=\{u, v\}, \phi(u)=1$ and $\phi(v)=2$ is given in the following corollary.

Corollary 4.22. Let $(j, l) \in A$ and $u, v \in I \backslash\{j, l\}$ such that $u \neq v$. Let $a=\max \left\{\left\lceil t_{j l}+t_{u l}+t_{v l}\right\rceil-\left\lceil t_{j l}+t_{u l}\right\rceil,\left\lceil t_{j l}+t_{v l}\right\rceil-\left\lceil t_{j l}\right\rceil\right\}$. Inequality

$$
\begin{aligned}
z_{j l} \geq & \left(\left\lceil t_{j l}+t_{v l}\right\rceil-a\right)\left(1-\sum_{m \in I \backslash\{j\}} x_{j m}\right)-\left\lceil t_{j l}+t_{u l}+t_{v l}\right\rceil \sum_{m \in I \backslash\{l\}} x_{l m} \\
& +\left(\left\lceil t_{j l}+t_{u l}+t_{v l}\right\rceil-\left\lceil t_{j l}+t_{v l}\right\rceil\right)\left(x_{u j}+x_{l u}\right)+a\left(x_{v j}+x_{l v}\right)
\end{aligned}
$$

is facet defining for $P_{j l}$.

Theorem 4.23. Let $(j, l) \in A, I^{\prime} \subseteq I \backslash\{j, l\}$ and $\phi$ be an order on $I^{\prime}$. For $i \in I^{\prime}$,

$$
\alpha_{i l}=-\left\lceil\sum_{m \in I^{\prime}} t_{j m}+t_{j l}\right\rceil+\left\lceil t_{j l}+\sum_{m \in I^{\prime} \backslash\{i\}} t_{j m}-\sum_{m \in I^{\prime}: \phi(m)<\phi(i)}\left(t_{j m}+\alpha_{m l}\right)^{+}\right\rceil .
$$


Inequality

$$
\begin{aligned}
z_{j l} \geq \sum_{i \in I^{\prime}} \alpha_{i l}(1- & \left.x_{i l}-x_{j i}-\sum_{m \in I \backslash\{l\}} x_{l m}\right) \\
+ & \left\lceil\sum_{m \in I^{\prime}} t_{j m}+t_{j l}\right]\left(1-\sum_{m \in I \backslash\{j\}} x_{j m}-\sum_{m \in I \backslash\{l\}} x_{l m}\right)
\end{aligned}
$$

is facet defining for $P_{j l}$.

Proof. The proof is analogous to the proof of Theorem 4.21.

Finally, using Theorems 4.14 and 4.15, we find the following facet defining inequalities.

Proposition 4.24. Let $(j, l) \in A$ and $u, v \in I \backslash\{j, l\}$ such that $u \neq v$. Let $a=\min \left\{\left\lceil t_{j l}+t_{j v}\right\rceil-\left\lceil t_{j l}\right\rceil,\left\lceil t_{j l}+t_{j v}+t_{u l}+t_{u v}\right\rceil-\left\lceil t_{j l}+t_{u l}\right\rceil\right\}$. Inequality

$$
\begin{aligned}
z_{j l} \geq & \left\lceil t_{j l}\right\rceil\left(1-\sum_{m \in I \backslash\{j\}} x_{j m}-\sum_{m \in I \backslash\{l\}} x_{l m}\right)+a\left(x_{v l}-\sum_{m \in I \backslash\{j, v\}} x_{j m}\right) \\
& +\left(\left\lceil t_{j l}+t_{u l}\right\rceil-\left\lceil t_{j l}\right\rceil\right)\left(x_{u j}-\sum_{m \in I \backslash\{l, u\}} x_{l m}\right)
\end{aligned}
$$

is facet defining for $P_{j l}$.

Proof. Inequality $z_{j l} \geq\left\lceil t_{j l}\right\rceil$ is facet defining for $P_{j l}(\emptyset)$. Now lift first $x_{u j}$ and then $x_{v l}$ using Theorems 4.14 and 4.15 , respectively. Inequality

$$
z_{j l} \geq\left\lceil t_{j l}\right\rceil+\left(\left\lceil t_{j l}+t_{u l}\right\rceil-\left\lceil t_{j l}\right\rceil\right) x_{u j}+a x_{v l}
$$

is facet defining for $P_{j l}(\{(u, j),(v, l)\})$. Next, by Theorem 4.11, optimal lifting coefficient for $x_{j m}$ with $m \in I \backslash\{j, v\}$ is $-\left\lceil t_{j l}\right\rceil-a$ and for $x_{j v}$ is $-\left\lceil t_{j l}\right\rceil$. The optimal coefficient of $x_{l m}$ for $m \in I \backslash\{l, u\}$ is $-\left\lceil t_{j l}+t_{u l}\right\rceil$ and for $x_{l u}$ is $-\left\lceil t_{j l}\right\rceil$.

Next, we lift variables $x_{i j}$ with $i \in I \backslash\{u, l, j\}$. As $x_{l j}$ is already lifted and for $m \in I \backslash\{i, j, l\}, x_{i m}$ and $x_{m i}$ are not lifted, condition set (ii) of Theorem 4.13 is satisfied and the lifting coefficient of $x_{i j}$ is zero.

For $x_{i l}$ with $i \in I \backslash\{v, j, l\}$, as $x_{j l}$ is already lifted and for $m \in I \backslash\{i, j, l\}, x_{i m}$ and $x_{m i}$ are not lifted, condition set (i) of Theorem 4.13 is satisfied. So lifting coefficient of $x_{i l}$ is zero.

Inequality (4.16) is facet defining for $P_{j l}\left(A^{\prime}\right)$, where $A^{\prime}=A \backslash\{(i, k): i \in I \backslash$ $\{j, l\}, k \in I \backslash\{i, j, l\}\}$. Next we lift $x_{i k}$ with $i \in I \backslash\{j, l\}$ and $k \in I \backslash\{i, j, l\}$. Optimal lifting coefficient is

$$
\alpha_{i k}=\min _{\left(x, z_{j l}\right) \in F_{j l}\left(A^{\prime} \cup\{(i, k)\}\right): x_{i k}=1} \sigma\left(x, z_{j l}\right),
$$

where

$$
\begin{aligned}
\sigma\left(x, z_{j l}\right)= & \left(z_{j l}-\left\lceil t_{j l}\right\rceil\left(1-\sum_{m \in I \backslash\{j\}} x_{j m}-\sum_{m \in I \backslash\{l\}} x_{l m}\right)-a\left(x_{v l}-\sum_{m \in I \backslash\{j, v\}} x_{j m}\right)\right. \\
& \left.-\left(\left\lceil t_{j l}+t_{u l}\right\rceil-\left\lceil t_{j l}\right\rceil\right)\left(x_{u j}-\sum_{m \in I \backslash\{l, u\}} x_{l m}\right)\right) .
\end{aligned}
$$


If $i \neq v$ and $k \neq v$, then let $x=e_{i k}^{x}+e_{j k}^{x}+e_{v l}^{x}$ and $z_{j l}=0$. If $i \neq v$ and $k=v$, then let $x=e_{i v}^{x}+e_{j v}^{x}$ and $z_{j l}=0$. If $i=v$ and $k \neq u$, then $x=e_{v k}^{x}+e_{l k}^{x}+e_{u j}^{x}$ and $z_{j l}=0$. Finally, if $i=v$ and $k=u$, then $x=e_{v u}^{x}+e_{l u}^{x}$ and $z_{j l}=0$. Solution $\left(x, z_{j l}\right) \in F_{j l}\left(A^{\prime} \cup\{(i, k)\}\right)$ with $x_{i k}=1$ and $\sigma\left(x, z_{j l}\right)=0$. We know by Proposition 4.10 that $\alpha_{i k} \geq 0$. Therefore, $\alpha_{i k}=0$. Repeating the same argument, we can prove that lifting coefficients of all variables $x_{i k}$ with $i \in I \backslash\{j, l\}$ and $k \in I \backslash\{i, j, l\}$ are zero.

An important issue is the separation of these inequalities. Inequalities (4.9), (4.10), (4.11), (4.14), and (4.16) can be separated in polynomial time by enumeration. The separation of inequalities (4.8), (4.12), (4.13), and (4.15) asks to choose a subset $I^{\prime} \subseteq I \backslash\{j, l\}$ and to find an order $\phi$ on $I^{\prime}$. We do not know the complexity of these problems.

5. Conclusion. In this paper, we presented polyhedral results for the HLM. By previous results, it was easy to characterize the facet defining inequalities that involve only the assignment or the capacity variables. It remained to investigate strong valid inequalities that involved both types of variables. We presented valid inequalities, results that give the optimal lifting coefficients of some variables as well as families of facet defining inequalities.

A future research direction is to study similar lifting results for $P_{B}$ where $B \subseteq A$ is not necessarily a singleton. Another one is to find efficient separation algorithms for the inequalities given here and incorporate these results in a branch and cut algorithm.

Acknowledgments. The author is grateful to an anonymous referee for his or her helpful comments on the structure and presentation and for drawing attention to several errors.

\section{REFERENCES}

[1] P. Avella And A. Sassano, On the p-median polytope, Math. Program., 89 (2001), pp. 395411.

[2] E. Balas and M. W. Padberg, Set partitioning: A survey, SiAM Rev., 18 (1976), pp. 710-760.

[3] J. F. Campbell, A. T. Ernst, and M. Krishnamoorthy, Hub location problems, in Facility Location: Applications and Theory, Z. Drezner and H. W. Hamacher, eds., Springer, New York, 2002, pp. 373-407.

[4] L. Cánovas, M. Landete, And A. Marín, Facet obtaining procedures for set packing problems, SIAM J. Discrete Math., 16 (2003), pp. 127-155.

[5] S. Chung, Y. Myung, And D. TCha, Optimal design of a distributed network with a two-level hierarchical structure, European J. Oper. Res., 62 (1992), pp. 105-115.

[6] H. W. Hamacher, M. Labbé, S. Nickel, and T. Sonneborn, Adapting polyhedral properties from facility to hub location problems, Discrete Appl. Math., 145 (2004), pp. 104-116.

[7] J. G. Klincewicz, Hub location in backbone/tributary network design: A review, Location Sci., 6 (1998), pp. 307-335.

[8] M. Labbé and H. Yaman, A Note on the Projection of Polyhedra, Preprint 2003/18, Université Libre de Bruxelles, 2003; also available online from http://www.ulb.ac.be/di/gom/ publications/technical/2003.html.

[9] M. Labbé and H. Yaman, Polyhedral Analysis for Concentrator Location Problems, Preprint 2003/13, Université Libre de Bruxelles, 2003; also available online from http://www.ulb.ac. be/di/gom/publications/technical/2003.html.

[10] M. Labbé and H. Yaman, Projecting the flow variables for hub location problems, Networks, 44 (2004), pp. 84-93.

[11] M. Labbé and H. Yaman, Solving the Uncapacitated Concentrator Location Problem with Star Routing, Preprint 2003/15, Université Libre de Bruxelles, 2003; also available online from http://www.ulb.ac.be/di/gom/publications/technical/2003.html.

[12] M. Labbé, H. Yaman, And E. Gourdin, A branch and cut algorithm for hub location problems with single assignment, in Math. Program., 102 (2005), pp. 371-405. 
[13] G. L. Nemhauser and L. A. Wolsey, Integer and Combinatorial Optimization, John Wiley, New York, 1988.

[14] M. W. PAdBerg, On the facial structure of set packing polyhedra, Math. Program., 5 (1973), pp. 199-215.

[15] H. Yaman and G. Carello, Solving the hub location problem with modular link capacities, Comput. Oper. Res., 32 (2005), pp. 3227-3245. 